Journal Club

Editor's Note: These short, critical reviews of recent papers in the Journal, written exclusively by graduate students or postdoctoral fellows, are intended to summarize the important findings of the paper and provide additional insight and commentary. For more information on the format and purpose of the Journal Club, please see http://www.jneurosci.org/misc/ifa_features.shtml.

\title{
Proteasomal Inhibition as a Treatment Strategy for Parkinson's Disease: The Impact of $\alpha$-Synuclein on Nurr 1
}

\author{
Michael J. Devine \\ Department of Molecular Neuroscience, Institute of Neurology, University College London, London WC1N 3BG, United Kingdom \\ Review of Lin et al.
}

\section{Introduction}

Parkinson's disease (PD) is a common and incurable neurodegenerative disorder. Loss of midbrain dopaminergic neurons (mDA) of the substantia nigra is the key pathological event in this disorder, but why this cell loss occurs remains a mystery. Much progress has been made by studying genes mutated in inherited forms of PD, thereby identifying cell pathways that might contribute to neuronal death (Hardy, 2010). One such gene is SNCA which encodes $\alpha$-synuclein. Point mutations and multiplications of SNCA cause familial PD, and variation at this locus is linked with the common sporadic form of the disease. Furthermore, $\alpha$-synuclein protein is the main component of Lewy bodies: cytoplasmic inclusions seen in mDA neurons that define the disease pathologically. Thus, $\alpha$-synuclein appears to play a central role in PD pathogenesis. However, studying how it exerts its toxicity has been hampered by the inaccessibility of diseased neurons from patients with the condition, and cell culture systems and transgenic animal models have generally failed to fully recapitulate the human disease.

Received Sept. 4, 2012; revised Sept. 26, 2012; accepted Sept. 27, 2012. This work was funded by a Clinical Research Training Fellowship from the Medical Research Council.

Correspondence should be addressed to Michael J. Devine, Department of Molecular Neuroscience, Institute of Neurology, University College London, London WC1N 3BG, United Kingdom. E-mail: michael.devine.09@ucl.ac.uk. DOI:10.1523/JNEUROSCI.4224-12.2012

Copyright $\odot 2012$ the authors $\quad$ 0270-6474/12/3216071-03\$15.00/0
In a recent paper in The Journal of Neuroscience, Lin et al. (2012) described a novel transgenic mouse that aims to model PD-related $\alpha$-synuclein pathogenesis more fully. First, expression of the tetracycline transactivator (tTA) was brought under control of the promoter of Pitx3, which is mainly expressed in $\mathrm{mDA}$ neurons from mid-gestation and throughout adulthood. These animals were crossed with a tetO-A53T line in which expression of human SNCA bearing the diseasecausing mutation A53T is triggered by the presence of tTA. This strategy yielded mice that express human A53T SNCA (hereafter A53T for short) selectively targeted to $\mathrm{mDA}$ neurons. A twofold to fourfold increase in expression over endogenous protein was observed.

These A53T mice displayed features in keeping with existing cell and animal models of PD. They had profound posture and motor abnormalities (impairments in rearing, gait and rotarod test) by 1 or 2 months of age, although they developed normally and had a normal life expectancy. Critically, compared with agematched wild-type littermates, they lost up to $40 \%$ of mDA neurons in the substantia nigra pars compacta and ventral tegmental area by 12 months of age. The length and complexity of neurons positive for tyrosine hydroxylase ( $\mathrm{TH}$, a component of the dopamine biosynthetic pathway and a marker of $\mathrm{mDA}$ neuronal identity) were reduced in A53T mice, and astrocytosis and microgliosis were present in the midbrain in these animals. Increased accumulation of $\alpha$-synuclein was seen in $\mathrm{mDA}$ neurons of A53T mice at both 12 and 18 months of age. Western blot analysis showed more highmolecular-weight bands positive for $\alpha$-synuclein in A53T tissue, suggesting that A53T mice have a greater proportion of aggregated $\alpha$-synuclein than in controls (which is pertinent to PD because Lewy bodies are largely comprised of aggregated $\alpha$-synuclein). Abnormalities in the Golgi, in synaptic release of dopamine, and in autophagy and lysosomal pathways were also detected.

Having revealed features consistent with PD pathology, Lin et al. (2012) sought to determine a mechanism for the loss of mDA neurons. The rearing abnormalities in the A53T mice provided a clue: the authors noted their similarity to those in a Nurr1 conditional knock-out mouse. Nurr1 controls the transcription of genes critical to mDA neurons, and is important for the maintenance of functional properties and survival of these cells. Progressive loss of mDA neurons occurs when Nurr1 is genetically ablated after terminal differentiation (Kadkhodaei et al., 2009), and Nurr1 expression is significantly decreased in mDA neurons in postmortem PD brain (Chu et al., 2006). Lin et al. (2012) asked whether $\alpha$-synuclein might be responsible for the reduction in Nurr1 by examining the expression and function of Nurr1 in A53T mice. Nurr1 expression was reduced in some $\mathrm{mDA}$ neurons, but unchanged in non-DA cells. Although not quantified, from cell to cell the presence of excess $\alpha$-synuclein appeared to correlate 
with a reduction of nuclear Nurr1, and affected cells had reduced expression of $\mathrm{TH}$ neuronal markers. In contrast, $\mathrm{TH}-$ positive neurons lacking $\alpha$-synuclein accumulation had normal Nurr1 staining.

These differences in Nurr1 staining were seen despite Nurr1 mRNA levels being comparable between A53T and wildtype mice, implying that modulation of Nurr1 by $\alpha$-synuclein occurs after transcription. This was examined in HEK293 cells, where transient expression of wildtype or A53T $\alpha$-synuclein was found to suppress expression of endogenous Nurr1, whereas Nurr1 expression increased following proteasomal inhibition using the selective inhibitor MG132. These findings were taken forward in primary neuronal cultures from A53T and wild-type mice. In these cells, five days of treatment with MG132 significantly increased nuclear Nurr1 staining in, and survival of, TH-positive neurons from A53T mice, but had no effect on control neurons. Lin et al. (2012) concluded that stabilization of Nurr1 via proteasomal inhibition may ameliorate degeneration of mDA neurons induced by $\alpha$-synuclein.

The conclusion that proteasomal inhibition might rescue neurons is surprising. The ubiquitin proteasome system is thought to play a critical role in maintaining cellular health by depletion of unwanted or misfolded proteins. Moreover, disruption of this system has been proposed to contribute to several neurodegenerative diseases, including PD. Although Lin et al. (2012) observed no clear change in proteasomal function in untreated A53T mice, function was impaired in previous PD models in which $\alpha$-synuclein was mutated or overexpressed (Cook et al., 2012). Furthermore, genetic ablation of the $26 \mathrm{~S}$ proteasome complex in transgenic mice, which completely abolishes ubiquitinmediated proteasomal degradation, causes neurodegeneration and intraneuronal $\alpha$-synuclein pathology resembling human Lewy bodies (Bedford et al., 2008). These models would predict that A53T neurons fair worse rather than better after administration of MG132.

How might these separate studies be reconciled? It is important to highlight their differences. For example, in contrast to the A53T mice used by Lin et al. (2012), previous transgenic models have generated substantial increases in $\alpha$-synuclein expression, far beyond the level seen in $\mathrm{PD}$, with potentially different effects on proteasomal function (Lin et al., 2009). The concentration of MG132 may also be pivotal. Lin et al. (2012) used a low MG132 concentration (10 nM) when treating neurons, whereas a higher

A Steady state

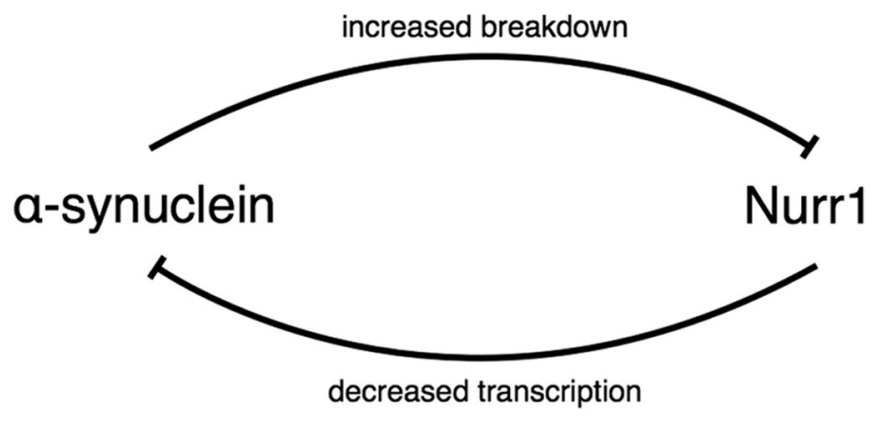

B Brief / partial proteasomal block

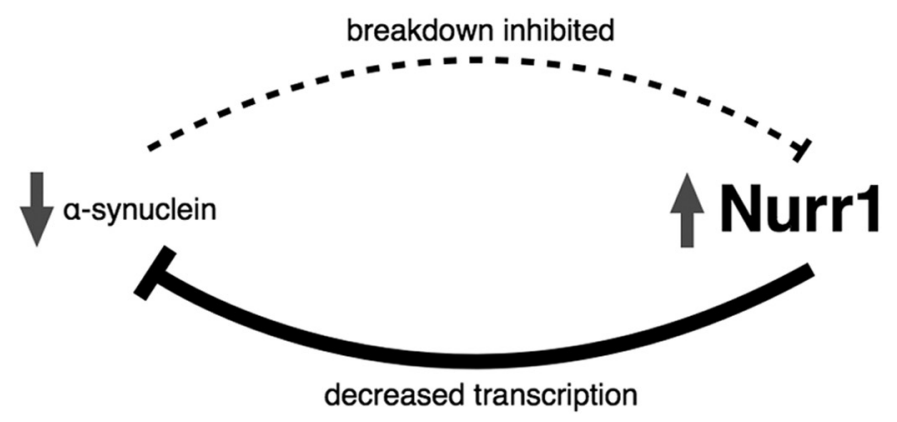

C Chronic / complete proteasomal block

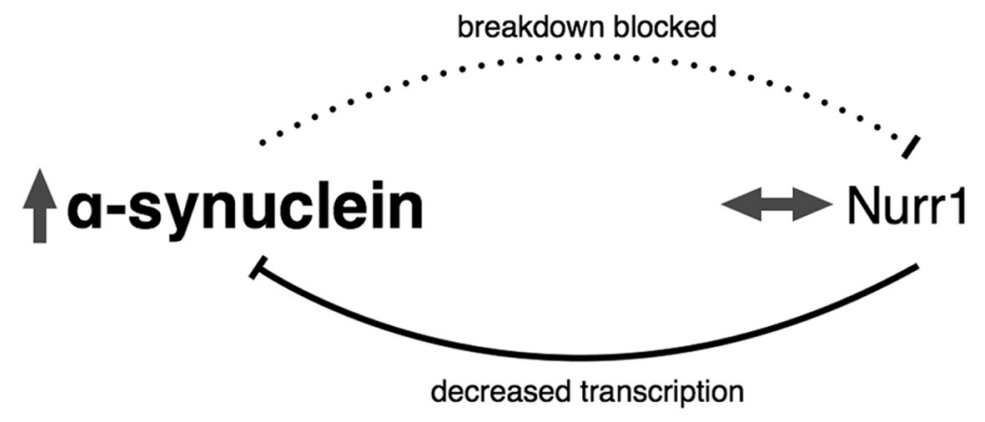

Figure 1. The relationship between Nurr1 and $\alpha$-synuclein under different proteasomal conditions. $\boldsymbol{A}$, Under steady-state conditions, each protein negatively regulates the abundance of the other. Nurr1 inhibits the transcription of $\alpha$-synuclein, while $\alpha$-synuclein expedites the proteasomal breakdown of Nurr1. $\boldsymbol{B}$, With brief, partial proteasomal inhibition, $\alpha$-synuclein loses its ability to negatively regulate Nurr1, but Nurr1 can still inhibit $\alpha$-synuclein. A positive feedback loop is created, favoring Nurr1. C, With chronic, complete proteasomal inhibition, existing models predict that $\alpha$-synuclein will accumulate, despite Nurr1 being relieved of negative regulation. This suggests that Nurr1 levels can be suppressed by other means or that Nurr 1 activity is impaired after $\alpha$-synuclein levels become too high.

concentration $(10 \mu \mathrm{M})$ was used to treat HEK293 cells. Different concentrations of MG132, in the range seen here, have opposite effects on virally induced apo- ptosis in a prostate cancer cell line: as inhibition is increased, proapoptotic factors accumulate and can outcompete anti-apoptotic factors, resulting in cell 
death (Lin et al., 1998). The time course of inhibition should also be noted: inhibition over a matter of hours in rat cerebellar neurons can be anti-apoptotic, whereas longer treatment $(16 \mathrm{~h})$ is proapoptotic (Butts et al., 2005). The fiveday treatment used by Lin et al. (2012) might have a different effect than the permanent inhibition seen in $26 \mathrm{~S}$ proteasome knock-out mice (Bedford et al., 2008).

There is a further point to consider. Lin et al. (2012) showed that $\alpha$-synuclein negatively regulates Nurr1, but Nurr1 has previously been shown to negatively regulate transcription of $\alpha$-synuclein in neuroblastoma cells (Yang and Latchman, 2008). Therefore, a positive feedback loop may exist, wherein a small increase in one of these two proteins can self-perpetuate (Fig. 1). Although this loop would undoubtedly be subject to complex regulation, it is conceivable that brief and partial proteasomal inhibition might favor accumulation of Nurr1, at the expense of $\alpha$-synuclein. As proteasomal inhibition becomes more complete, and of longer duration, accumulation of $\alpha$-synuclein may dominate Nurr1 and evade Nurr1-mediated transcriptional repression. However, under these conditions the prediction would be that Nurrl levels would be maintained to some extent, perhaps partially offsetting neuronal death induced by $\alpha$-synuclein.

It would be intriguing to examine the effect of a longer duration or higher dose of MG132 treatment on A53T neurons. What would happen to Nurr1 levels under these circumstances? If depleted, this would suggest that $\alpha$-synuclein can sup- press Nurr1 levels via other mechanisms besides proteasomal regulation. If not, perhaps $\alpha$-synuclein can impair Nurr 1 activity directly. Although demonstrated in primary neuronal cultures, it would be of great importance to determine whether proteasomal inhibition via MG132 (or another inhibitor) can rescue A53T neurons in vivo. The A53T mouse model could be used to determine the therapeutic window for proteasomal inhibition in the treatment of $\alpha$-synuclein toxicity, if indeed such a window exists.

The study by Lin et al. (2012) points to a novel disease pathway in PD, whereby $\alpha$-synuclein depletes vulnerable neurons of proteins important for survival, and the study provides thought-provoking data on the possible role of proteasomal inhibition in the treatment of this disorder. Proteasomal inhibition is generally regarded as having a detrimental impact upon neuronal health in PD, but Lin et al. (2012) show that the interaction between key pathogenic proteins and the proteasome is likely to be much more nuanced than previously thought.

\section{References}

Bedford L, Hay D, Devoy A, Paine S, Powe DG, Seth R, Gray T, Topham I, Fone K, Rezvani N, Mee M, Soane T, Layfield R, Sheppard PW, Ebendal T, Usoskin D, Lowe J, Mayer RJ (2008) Depletion of $26 \mathrm{~S}$ proteasomes in mouse brain inhibition versus induction of apoptosis by proteasome inhibitors depends on concentration. J Neurosci 28:8189-8198.

Butts BD, Hudson HR, Linseman DA, Le SS, Ryan KR, Bouchard RJ, Heidenreich KA (2005) Proteasome inhibition elicits a biphasic effect on neuronal apoptosis via differential regulation of pro-survival and pro-apoptotic transcription factors. Mol Cell Neurosci 30:279-289.

Chu Y, Le W, Kompoliti K, Jankovic J, Mufson EJ, Kordower JH (2006) Nurrl in Parkinson's disease and related disorders. J Comp Neurol 494:495-514.

Cook C, Stetler C, Petrucelli L (2012) Disruption of protein quality control in Parkinson's disease. Cold Spring Harb Perspect Med 2:a009423.

Hardy J (2010) Genetic analysis of pathways to Parkinson disease. Neuron 68:201-206.

Kadkhodaei B, Ito T, Joodmardi E, Mattsson B, Rouillard C, Carta M, Muramatsu S, SumiIchinose C, Nomura T, Metzger D, Chambon P, Lindqvist E, Larsson NG, Olson L, Björklund A, Ichinose H, Perlmann T (2009) Nurr1 is required for maintenance of maturing and adult midbrain dopamine neurons. J Neurosci 29:15923-15932.

Lin KI, Baraban JM, Ratan RR (1998) Inhibition versus induction of apoptosis by proteasome inhibitors depends on concentration. Cell Death Differ 5:577-583.

Lin X, Parisiadou L, Gu XL, Wang L, Shim H, Sun L, Xie C, Long CX, Yang WJ, Ding J, Chen ZZ, Gallant PE, Tao-Cheng JH, Rudow G, Troncoso JC, Liu Z, Li Z, Cai H (2009) Leucinerich repeat kinase 2 regulates the progression of neuropathology induced by Parkinson'sdisease-related mutant $\alpha$-synuclein. Neuron 64:807-827.

Lin X, Parisiadou L, Sgobio C, Liu G, Yu J, Sun L, Shim H, Gu XL, Luo J, Long CX, Ding J, Mateo Y, Sullivan PH, Wu LG, Goldstein DS, Lovinger D, Cai H (2012) Conditional expression of Parkinson's disease-related mutant $\alpha$-synuclein in the midbrain dopaminergic neurons causes progressive neurodegeneration and degradation of transcription factor nuclear receptor related 1 . J Neurosci 32:9248-9264.

Yang YX, Latchman DS (2008) Nurrl transcriptionally regulates the expression of alphasynuclein. Neuroreport 19:867-871. 